Brit. J. vener. Dis. (1956), 32, 109.

\title{
A COMPARATIVE STUDY OF PRICE'S PRECIPITATION REACTION (PPR) USING OX AND GOAT ANTIGENS
}

\author{
BY \\ S. A. KHAN \\ Central Skin and Social Hygiene Centre, Karachi
}

When the WHO (VD) demonstration team left Karachi in July 1954, the routine serological tests for syphilis (STS) performed in the centre were the Price Precipitation Reaction (PPR) and the Slide test (VDRL). The team left a fair quantity of "Original Extract MKR 11 " antigen and, to avoid wastage, the Meinicke test was included among the routine tests of the centre.

The antigen for the PPR was prepared from ox heart in the laboratory according to the standard technique (Price, 1948).

To explore the possibility of preparing antigen from the heart muscle of animals other than the ox, which could produce results parallel to the PPR, the hearts of sheep, goats, cows, and buffaloes were obtained. Initially, 40 per cent. $\mathrm{w} / \mathrm{v}$ alcoholic extracts of heart muscle were used for antigen preparation. This higher concentration was used in preference to the 20 per cent. extraction employed in the original description of the PPR because the 40 per cent. solution could be diluted until the results conformed with the PPR (ox heart) in sensitivity and specificity. The antigens from the heart muscles of the buffalo, sheep, and cow were not sensitive enough, but with goat's heart antigen the results were very encouraging. However, the goat heart antigen occasionally was not sensitive to weak positive sera and, believing that 40 per cent. solution contained too high a concentration of lipoids, the percentage was gradually reduced. A 20 per cent. solution gave results approximately similar to those obtained with the ox heart antigen.

Before use in the test proper, the precipitation titre was determined by the method used for ox heart PPR antigen. The titre was usually found to be $0 \cdot 6$, but occasionally $0 \cdot 8$. To prepare the antigen emulsion for the test, $1 \mathrm{ml}$. of the alcoholic extract was mixed with 0.6 or $0.8 \mathrm{ml}$. of saline according to the titre and after standing and centrifuging for

\footnotetext{
* Received for publication November 21, 1955.
}

10 minutes, the deposit was resuspended in $0.6 \mathrm{ml}$. saline. Once the titre of a batch of alcoholic extract of goat heart muscle had been determined, it remained constant, as is found with antigen prepared from ox heart.

During the testing of various batches, antigen from male hearts was found to be more reactive than that from female hearts. Testicular and brain alcoholic extracts were added to goat antigen in small quantities, but this did not improve the results. In the screen test the goat antigen often gave positive results where the ox antigen gave negative results caused by prozone phenomena. When the quantitative PPR was performed on the same sera they gave positive results. Sera which were positive with goat antigen were usually also positive to Meinicke and VDRL tests.

Altogether 7,211 PPRs were performed on sera received from the centre. The cases of venereal disease included fresh sores, gonorrhoea, and all stages of syphilis. The results with PPR (ox) and PPR (goat) were similar with a variation of only 0.75 per cent. among the positive cases.

The details are shown in the correlation square :

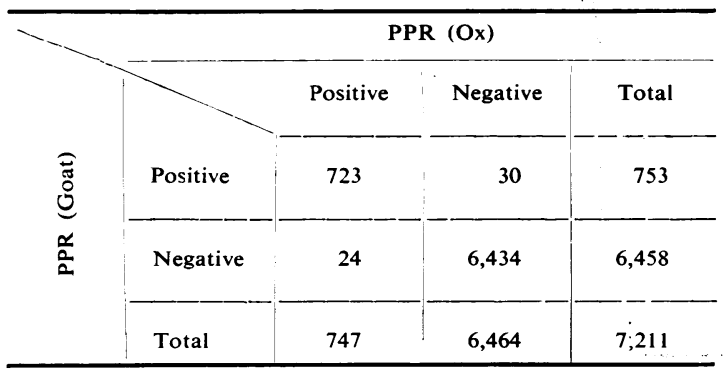

Table I (overleaf) includes thirty cases which were PPR (ox) negative but PPR (goat) and VDRL positive. More than half of these cases were also positive with the Meinicke test, which was performed on active sera, if available after routine test. 
TABLE I

COMPARATIVE STUDY OF PPR OX AND GOAT ANTIGENS PPR OX NEGATIVE AND GOAT POSITIVE

\begin{tabular}{|c|c|c|c|c|c|c|c|c|}
\hline \multirow{2}{*}{$\begin{array}{c}\text { Type of } \\
\text { Case }\end{array}$} & \multirow{2}{*}{$\begin{array}{l}\text { Case } \\
\text { No. }\end{array}$} & Diagnosis & \multirow{2}{*}{$\begin{array}{c}\text { History } \\
\text { of } \\
\text { Venereal } \\
\text { Disease }\end{array}$} & \multicolumn{2}{|c|}{ PPR } & \multirow{2}{*}{$\begin{array}{l}\text { VDRL } \\
\text { Positive }\end{array}$} & \multicolumn{2}{|c|}{ Meinicke } \\
\hline & & Type of Syphilis & & $\mathbf{O x}$ & Goat & & Rapid & $\begin{array}{l}\text { Over- } \\
\text { night }\end{array}$ \\
\hline $\begin{array}{l}\text { Fresh } \\
\text { Cases }\end{array}$ & $\begin{array}{r}1 \\
2 \\
3 \\
4 \\
5 \\
6 \\
7 \\
8 \\
9 \\
10 \\
11 \\
12 \\
13 \\
14\end{array}$ & $\begin{array}{l}\text { Sero-positive, tertiary : bones and joints } \\
\text { Sero-positive, tertiary latent, more than } 4 \text { years } \\
\text { Untreated congenital } \\
\text { Parasyphilis } \\
\text { Sero-positive, primary } \\
\text { Parasyphilis } \\
\text { Sero-positive, tertiary : skin and mucous membranes } \\
\text { Parasyphilis } \\
\text { Sero-positive, secondary : less than } 6 \text { months } \\
\text { Parasyphilis } \\
\text { Sero-negative, primary } \\
\text { Parasyphilis } \\
\text { Parasyphilis } \\
\text { Sero-positive, tertiary }\end{array}$ & $\begin{array}{l}\text { Yes } \\
\text { Yes } \\
\text { Yes } \\
\text { Yes } \\
\text { Yes } \\
\text { Yes } \\
\text { Yes } \\
\text { Yes } \\
\text { Yes } \\
\text { Yes } \\
\text { Yes } \\
\text { Yes } \\
\text { Yes } \\
\text { Yes }\end{array}$ & $\begin{array}{l}\text { Negative } \\
\text { Negative } \\
\text { Negative } \\
\text { Negative } \\
\text { Negative } \\
\text { Negative } \\
\text { Negative } \\
\text { Negative } \\
\text { Negative } \\
\text { Negative } \\
\text { Negative } \\
\text { Negative } \\
\text { Negative } \\
\text { Negative }\end{array}$ & $\begin{array}{l}\text { Neat } \\
\text { Positive 2 } \\
\text { Positive } 64 \\
\text { Positive } 4 \\
\text { Neat } \\
\text { Neat } \\
\text { Positive 2 } \\
\text { Positive 2 } \\
\text { Neat } \\
\text { Neat } \\
\text { Neat } \\
\text { Neat } \\
\text { Neat } \\
\text { Neat }\end{array}$ & $\begin{array}{r}4 \\
4 \\
256 \\
4 \\
4 \\
8 \\
8 \\
8 \\
4 \\
4 \\
8 \\
2 \\
8 \\
8\end{array}$ & $\begin{array}{l}\text { ** } \\
* \\
* \\
* \\
* * \\
- \\
* \\
* \\
* \\
\\
*\end{array}$ & $\begin{array}{l}* * \\
* * \\
* * * \\
* \\
* * \\
- \\
* \\
* \\
* \\
* \\
*\end{array}$ \\
\hline $\begin{array}{c}\text { Treated } \\
\text { Cases }\end{array}$ & $\begin{array}{l}15 \\
16 \\
17 \\
18 \\
19 \\
20 \\
21 \\
22 \\
23 \\
24 \\
25 \\
26 \\
27 \\
28 \\
29 \\
30\end{array}$ & $\begin{array}{l}\text { Sero-positive, tertiary : skin and mucous membranes } \\
\text { Sero-positive, latent untreated } \\
\text { Sero-positive, tertiary latent : more than } 4 \text { years } \\
\text { Sero-positive, secondary : less than } 6 \text { months } \\
\text { Parasyphilis } \\
\text { Sero-positive, tertiary latent : more than } 4 \text { years } \\
\text { Sero-positive, tertiary latent : more than } 4 \text { years } \\
\text { Sero-positive, secondary: less than } 6 \text { months } \\
\text { Condylomata acuminata } \\
\text { Pruritus } \\
\text { Impetigo } \\
\text { Xerodermia } \\
\text { Ulcer on forearm } \\
\text { Tinea } \\
\text { Traumatic ulcer } \\
\text { Impetigo }\end{array}$ & $\begin{array}{l}\text { Yes } \\
\text { Yes } \\
\text { Yes } \\
\text { Yes } \\
\text { Yes } \\
\text { Yes } \\
\text { Yes } \\
\text { Yes } \\
\text { No } \\
\text { No } \\
\text { No } \\
\text { No } \\
\text { No } \\
\text { No } \\
\text { No } \\
\text { No }\end{array}$ & $\begin{array}{l}\text { Negative } \\
\text { Negative } \\
\text { Negative } \\
\text { Negative } \\
\text { Negative } \\
\text { Negative } \\
\text { Negative } \\
\text { Negative } \\
\text { Negative } \\
\text { Negative } \\
\text { Negative } \\
\text { Negative } \\
\text { Negative } \\
\text { Negative } \\
\text { Negative } \\
\text { Negative }\end{array}$ & $\begin{array}{l}\text { Neat } \\
\text { Neat } \\
\text { Neat } \\
\text { Neat } \\
\text { Neat } \\
\text { Neat } \\
\text { Neat } \\
\text { Neat } \\
\text { Neat } \\
\text { Neat } \\
\text { Positive } \\
\text { Neat } \\
\text { Neat } \\
\text { Neat } \\
\text { Neat } \\
\text { Neat }\end{array}$ & \begin{tabular}{|c|}
4 \\
8 \\
8 \\
4 \\
2 \\
16 \\
8 \\
8 \\
4 \\
2 \\
Positive \\
4 \\
16 \\
8 \\
4 \\
4
\end{tabular} & $\begin{array}{c}\bar{Z} \\
\bar{*} \\
\bar{*} \\
\bar{*} \\
\text { Negative } \\
\bar{*} \\
\overline{-} \\
\overline{-}\end{array}$ & 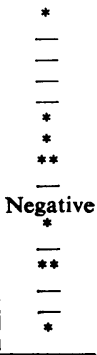 \\
\hline
\end{tabular}

Neat $=$ Positive with undiluted serum. $\quad$ Meinicke Tests : * Weak positive. ** Positive. *** Strong positive.

- No serum for test.

TABLE II

COMPARATIVE STUDY OF PPR OX AND GOAT ANTIGENS

PPR OX POSITIVE AND GOAT NEGATIVE

\begin{tabular}{|c|c|c|c|c|c|c|c|c|}
\hline \multirow{2}{*}{$\begin{array}{c}\text { Type of } \\
\text { Case }\end{array}$} & \multirow{2}{*}{$\begin{array}{l}\text { Case } \\
\text { No. }\end{array}$} & Diagnosis & \multirow{2}{*}{$\begin{array}{c}\text { History } \\
\text { of } \\
\text { Venereal } \\
\text { Disease }\end{array}$} & \multicolumn{2}{|c|}{ PPR } & \multirow{2}{*}{$\frac{\text { VDRL }}{\text { Positive }}$} & \multicolumn{2}{|c|}{ Meinicke } \\
\hline & & Type of Syphilis & & $\mathbf{O x}$ & Goat & & Rapid & $\begin{array}{l}\text { Over- } \\
\text { night }\end{array}$ \\
\hline $\begin{array}{l}\text { Fresh } \\
\text { Cases }\end{array}$ & $\begin{array}{l}31 \\
32 \\
33 \\
34 \\
35 \\
36 \\
37 \\
38 \\
39 \\
40 \\
41\end{array}$ & $\begin{array}{l}\text { Sero-positive, latent untreated } \\
\text { Sero-positive, tertiary latent : more than } 4 \text { years } \\
\text { Sero-positive, latent untreated } \\
\text { Parasyphilis } \\
\text { Sero-positive, tertiary latent : more than } 4 \text { years } \\
\text { Sero-positive, latent untreated } \\
\text { Sero-positive, latent untreated } \\
\text { Sero-positive, latent untreated } \\
\text { Sero-positive, tertiary : optic atrophy } \\
\text { Latent } \\
\text { Congenital other than visual }\end{array}$ & $\begin{array}{l}\text { Yes } \\
\text { Yes } \\
\text { Yes } \\
\text { Yes } \\
\text { Yes } \\
\text { Denied } \\
\text { Yes } \\
\text { Denied } \\
\text { Denied } \\
\text { Denied } \\
\text { Denied }\end{array}$ & $\begin{array}{l}\text { Neat } \\
\text { Neat } \\
\text { Neat } \\
\text { Positive } 2 \\
\text { Positive } 2 \\
\text { Neat } \\
\text { Neat } \\
\text { Positive } 4 \\
\text { Positive } 64 \\
\text { Positive } 2 \\
\text { Neat }\end{array}$ & $\begin{array}{l}\text { Negative } \\
\text { Negative } \\
\text { Negative } \\
\text { Negative } \\
\text { Negative } \\
\text { Negative } \\
\text { Negative } \\
\text { Negative } \\
\text { Negative } \\
\text { Negative } \\
\text { Negative }\end{array}$ & $\begin{array}{r}4 \\
8 \\
64 \\
-8 \\
16 \\
16 \\
8 \\
64 \\
8 \\
16\end{array}$ & $\begin{array}{l}* * \\
Z \\
= \\
= \\
* \\
* * \\
* * \\
* \\
*\end{array}$ & $\begin{array}{c}* * \\
* \\
* * * \\
* * * \\
* \\
* \\
* \\
* * \\
* \\
* *\end{array}$ \\
\hline $\begin{array}{c}\text { Treated } \\
\text { Cases }\end{array}$ & $\begin{array}{l}42 \\
43 \\
44 \\
45 \\
46 \\
47 \\
48 \\
49 \\
50 \\
51 \\
52 \\
53 \\
54\end{array}$ & $\begin{array}{l}\text { Sero-positive, tertiary : skin and mucous membranes } \\
\text { Sero-positive, tertiary latent : more than } 4 \text { years } \\
\text { Sero-positive, secondary: less than } 6 \text { months } \\
\text { Sero-positive, secondary : less than } 6 \text { months } \\
\text { Sero-positive, primary } \\
\text { Sero-positive, tertiary : skin and mucous membranes } \\
\text { Sero-positive, tertiary latent : more than } 4 \text { years } \\
\text { Sero-positive, tertiary latent : more than } 4 \text { years } \\
\text { Sero-positive, tertiary latent : more than } 4 \text { years } \\
\text { Parasyphilis } \\
\text { Sero-positive, latent untreated } \\
\text { Sero-positive, tertiary latent : more than } 4 \text { years } \\
\text { Impetigo }\end{array}$ & $\begin{array}{l}\cdots \\
\cdots \\
\cdots \\
\cdots \\
\cdots \\
\cdots \\
\cdots \\
\cdots \\
\text { Denied }\end{array}$ & $\begin{array}{l}\text { Neat } \\
\text { Neat } \\
\text { Positive } 32 \\
\text { Positive } 2 \\
\text { Neat } \\
\text { Positive } 2 \\
\text { Neat } \\
\text { Neat } \\
\text { Positive } 2 \\
\text { Neat } \\
\text { Positive } 2 \\
\text { Positive } 2 \\
\text { Neat }\end{array}$ & $\begin{array}{c}\text { Negative } \\
\text { Negative } \\
\text { Negative } \\
\text { Negative } \\
\text { Negative } \\
\text { Negative } \\
\text { Negative } \\
\text { Negative } \\
\text { Negative } \\
\text { Negative } \\
\text { Negative } \\
\text { Negative } \\
\text { Negative }\end{array}$ & $\begin{array}{c}4 \\
16 \\
32 \\
8 \\
8 \\
2 \\
4 \\
4 \\
16 \\
\text { Positive } \\
16 \\
4 \\
-\end{array}$ & $\begin{array}{c}* * \\
* * \\
- \\
* \\
* \\
* \\
* * \\
* * * \\
* \\
* * * \\
* \\
-\end{array}$ & $\begin{array}{l}* * \\
* \\
* \\
-* \\
* * \\
* \\
* \\
* \\
* \\
* \\
* \\
-\end{array}$ \\
\hline
\end{tabular}

Neat $=$ Positive with undiluted serum. $\quad$ Meinicke Tests : * Weak positive. ** Positive. *** Strong positive.

- No serum for test.

Fourteen new cases (Cases 1-14) out of thirty were diagnosed as syphilis, as their sera were positive to the VDRL test, and the patients gave a history of a venereal sore. Eight cases (Cases 15-22) were on routine tests after completion of treatment. The remaining eight (Cases 23-30) denied exposure to 
risk, but were positive to VDRL and PPR (goat) ; three of them were also positive to the Meinicke test.

Table II (opposite) gives details of 24 PPR (ox) positive and PPR (goat) negative cases, which, with two exceptions, were also positive to VDRL and to the Meinicke test. These include eleven new cases (Cases 31-41) which were diagnosed as syphilis on the findings of the PPR although five of them denied exposure. Case 34 was positive to the PPR but negative to VDRL and the Meinicke test, but gave a history of a venereal sore. Twelve cases (Cases 42-53), which were positive to PPR (ox) but negative to PPR (goat), were treated cases who were attending for routine blood tests. Case 54 had impetigo, and the positive PPR but negative VDRL and Meinicke test was taken to be a biological false positive result.

It has been found that whereas antigen emulsion of PPR ox heart remains in active condition for a week at the most, the goat antigen emulsion remains active for up to $2 \frac{1}{2}$ weeks and gives results similar to those obtained with freshly prepared ox antigen.

\section{Summary}

(1) Altogether 7,211 sera were tested with PPR (ox) and PPR (goat) antigens and the results compared. The variation in the results was only 0.75 per cent.

(2) Their sensitivity and specificity were found to be alike after comparative studies using the VDRL, PPR, and Meinicke tests.

(3) In Pakistan, goat antigen may be used in place of ox antigen in PPR tests.

Thanks are due to the Director General of Health (Pakistan) for granting permission to do the tests and to the Director of the Central Skin and Social Hygiene Centre, Karachi, for providing facilities and material for the tests.

\section{REFERENCE}

Price, I. N. O. (1948). J. clin. Path., 1, 91. 